\title{
Predictors of improved functional outcome in elderly inpatients after rehabilitation: a retrospective study
}

\author{
Koji Naruishi ${ }^{1,2}$ \\ Akiko Kunita $^{3}$ \\ Katsuyuki Kubo ${ }^{1,2}$ \\ Toshihiko Nagata ${ }^{4}$ \\ Shogo Takashiba ${ }^{2,5}$ \\ Seiji Adachi' \\ 'Division of Local Care Center, \\ Tottori Municipal Hospital, Tottori, \\ Japan; ${ }^{2}$ Department of Periodontics \\ and Endodontics, Okayama University \\ Hospital, Okayama, Japan; ${ }^{3}$ Division \\ of Rehabilitation, Tottori Municipal \\ Hospital, Tottori, Japan; ${ }^{4}$ Department \\ of Periodontology and Endodontology, \\ Institute of Health Biosciences, \\ Tokushima University Graduate \\ School, Tokushima, Japan; ${ }^{5}$ Department \\ of Pathophysiology-Periodontal \\ Science, Okayama University \\ Graduate School of Medicine, \\ Dentistry and Pharmaceutical \\ Sciences, Okayama, Japan
}

This article was published in the following Dove Press journal:

Clinical Interventions in Aging

5 December 2014

Number of times this article has been viewed

Purpose: The number of elderly inpatients has been steadily increasing worldwide. However, the ability to predict the degree of improvement of functional capacity after comprehensive examination of elderly inpatients is still lacking. The purpose of this study was to investigate the predictors of improved functional outcome after rehabilitation of elderly inpatients.

Methods: We performed a retrospective cohort study with 1,079 patients (age $<70$ years: $\mathrm{N}=331$, age $\geq 70$ years: $\mathrm{N}=748$ ) who had been admitted to Tottori Municipal Hospital. Functional Independence Measure (FIM) scores were measured both at admission and discharge to calculate FIM gain and efficiency. Of these patients, 262 patients had oral examinations on admission. The Mann-Whitney $U$-test or chi-square test was used for statistical analyses. Conditional logistic regression analysis was used to compute the odds ratio (OR) and $95 \%$ confidence interval (CI). Cut-off values of FIM scores to determine if elderly inpatients were able to return home after discharge were determined using a receiver operating characteristic curve.

Results: FIM scores, including FIM gain and efficiency, of elderly patients were significantly lower than those of middle-aged patients. Inability to close the lips and dysfunctional tongue movement, but not the loss of teeth, were correlated with a reduced improvement of FIM scores. Cognitive impairment and aspiration pneumonia, but not cerebrovascular disease, were also correlated with a reduced improvement of FIM scores. Interestingly, FIM scores were significantly lower in patients with both cerebrovascular disease and a loss of posterior occlusion. Factors shown to have a significant impact on the improvement of FIM scores included the stable posterior occlusion (OR: 2.23, 95\% CI: 1.2-4.1), closed lips (OR: 5.15, 95\% CI: 2.3-11.7), functional tongue movement (OR: 5.74, 95\% CI: 3.0-11.0), presence of cognitive impairment (OR: 0.31, 95\% CI: 0.17-0.49), and presence of aspiration pneumonia (OR: $0.27,95 \%$ CI: $0.15-0.51$ ).

Conclusion: Age and disorder of oral function may be significant predictors of improved functional capacity after rehabilitation for elderly inpatients.

Keywords: elderly inpatients, Functional Independence Measure (FIM), oral examination, geriatric disease

\section{Introduction}

Japan is a steadily aging society, and now more so with the post-world war II baby boom generation reaching old age. ${ }^{1}$ Japan's population continues to age rapidly; while in 1950 , only $5 \%$ of the Japanese population was aged 65 years or older, the proportion increased to $7 \%$ in 1970 and then $23 \%$ in $2010 .{ }^{1}$ Elderly people are considered vulnerable members of society with regard to health, and the number of hospitalized elderly patients has been steadily increasing. An increase of the elderly population may weaken the country's economic strength, and it may also force changes in the framework of social welfare, such as medical expenses. ${ }^{1,2}$
Correspondence: Koji Naruishi Department of Periodontics, Tokushima University Hospital, 2-50-I Kuramoto,

Tokushima 770-8503, Japan

Tel +8I 88 63 I 3III

Fax +8I 886337009

Email naruishi@tokushima-u.ac.jp 
An extremely elderly person, unable to keep up with daily activities and maintenance, may gradually develop an unhealthy and debilitating living condition that may affect the progression of geriatric diseases, such as cerebrovascular disease, cognitive impairment, and aspiration pneumonia. ${ }^{3,4}$ Despite the immense impact of aging at a personal and societal level, the ability to predict the improvement of functional capacity of elderly inpatients after rehabilitation is still lacking. Ideally, elderly inpatients are rehabilitated by a physiatrist to improve the patient's functional capacity and outcome during the hospital stay, and then later discharged back to their homes. ${ }^{5}$ Through proper inpatient rehabilitation, almost all elderly inpatients should be able to recover and satisfactorily perform activities of daily living (ADLs). Nakayama et al has reported recently that there are significant relations between recovery of swallowing function and ADLs in stroke patients. ${ }^{6}$ On the other hand, in a few cases, elderly patients are unable to fully recover and perform ADLs even after undergoing the same methods of rehabilitation; this underlying reason has yet to be identified. ${ }^{7}$

Physical disability of the elderly manifests as the loss of ability to complete basic ADLs needed to live independently without assistance, such as bathing, dressing, rising from a bed or chair, using the toilet, eating, or walking across a room. ${ }^{8}$ The Functional Independence Measure (FIM) motor scale is used clinically to measure the patient's progress and assess rehabilitation outcomes. ${ }^{9,10}$ The FIM score was carefully designed and developed with the consensus of the US National Advisory Committee, with close attention to definitions, administration, and reliability. ${ }^{11}$

The present study focused on elderly patients who received comprehensive inpatient rehabilitation services while in the hospital. In order to improve the ability of clinicians to predict the functional outcome of elderly inpatients, we analyzed several factors such as age, sex, nutritional status, and oral condition and their association with the improvement of physical function.

\section{Materials and methods Subjects and evaluation}

A total of 1,079 patients admitted to the Tottori Municipal Hospital between January 2013 and December 2013 was enrolled in this study (age $<70$ years: $\mathrm{N}=331$, male/female: 180/151, average: $53.9 \pm 14.1$ years; age $\geq 70$ years: $\mathrm{N}=748$, male/female: $270 / 478$, average: $82.9 \pm 6.8$ years). All patients had received standard rehabilitation treatment by attending physicians, including range-of-motion exercises, resistance training, physical restoration, movement exercises, and ambulation exercises during their hospital stay. Physical ability of the patients was assessed using FIM scores at admission and again at discharge. FIM gain was calculated as the change in FIM score at hospital discharge. FIM efficiency was calculated by subtracting FIM at admission from FIM at discharge and then dividing by the length of stay in days. Of 1,079 patients, the oral conditions of 262 elderly patients (male/female: 101/161, average age: male, $83.2 \pm 6.6$ years, female, $85.9 \pm 6.0$ years) were evaluated by inspection and palpation, including stability of the posterior occlusion, ability to close the lips, tongue movement, absence of teeth, and presence of mobile teeth. For posterior occlusion stability determination, we defined inpatients with normal posterior teeth or dentures as "stable". Nutritional status was evaluated by body mass index (BMI). FIM was evaluated by physical therapists, oral condition was evaluated by trained dentists, and nutritional status was evaluated by nurses, blinded to the patient's conditions. Cognitive impairment of elderly inpatients was evaluated using the clinical dementia rating scale. ${ }^{12}$ This study was approved by the ethics committee of Tottori Municipal Hospital (No 1153). We did not obtain written informed consent because guidelines for epidemiological research established by the Japanese Ministry of Health, Labour and Welfare do not require written informed consent for retrospective studies.

\section{Statistical analysis}

Statistical analyses were performed using JMP ${ }^{\circledR} 8$ version 8.0.2 (SAS Institute Inc., Cary, NC, USA). Data were described by the mean \pm standard deviation. Correlation between FIM scores at admission and discharge with oral condition, BMI, and presence of diseases such as cerebrovascular disease, cognitive impairment, and aspiration pneumonia was calculated. Because FIM scores were not normally distributed, the Mann-Whitney $U$-test and Spearman's rank correlation coefficient were used for statistical analysis. Differences in residence between the time of discharge and admission of patients aged either under or over 70 years were analyzed by chi-square test. The odds ratios (ORs) derived from a bivariate analysis and $95 \%$ confidence intervals (CIs) of the improvement of FIM scores ( $>10$ points) with regard to oral conditions were obtained by conditional logistic regression analysis. In all statistical analyses, $P$-values $<0.05$ were considered statistically significant.

\section{Results \\ Living situation of elderly inpatients compared to middle-aged inpatients}

Differences between elderly and middle-aged inpatients are shown in Table 1. The length of hospital stay of elderly 
Table I Comparison of each age group in hospital stay

\begin{tabular}{|c|c|c|c|}
\hline & Below 70 yr & Above $70 \mathrm{yr}$ & $P$-value \\
\hline$N(M / F)$ & $331(180 / I 5 I)$ & $748(270 / 478)$ & \\
\hline Age $(y r)$ & $53.9 \pm \mid 4.1$ & $82.9 \pm 6.8$ & $<0.0001 *$ \\
\hline $\begin{array}{l}\text { Length of } \\
\text { hospital stay (day) }\end{array}$ & $28.9 \pm 27.7$ & $33.4 \pm 24.4$ & $<0.000 I^{*}$ \\
\hline \multicolumn{4}{|l|}{ FIM score } \\
\hline At admission & $82.2 \pm 25.3$ & $56.0 \pm 26.2$ & $<0.0001 *$ \\
\hline At discharge & $108.0 \pm 23.9$ & $76.6 \pm 34.9$ & $<0.0001 *$ \\
\hline FIM gain & $25.8 \pm 23.3$ & $20.6 \pm 21.5$ & $<0.0001 *$ \\
\hline FIM efficiency & $1.44 \pm 1.5$ & $0.83 \pm 1.0$ & $<0.000$ I* \\
\hline \multicolumn{4}{|c|}{ Residence at admission } \\
\hline Home & 313 & 568 & $<0.0001 * *$ \\
\hline Hospital $^{\dagger}$ & 18 & 180 & \\
\hline \multicolumn{4}{|c|}{ Residence at discharge } \\
\hline Home & 287 & 445 & $<0.0001 * *$ \\
\hline Hospital $^{\dagger}$ & 38 & 280 & \\
\hline Dead & 6 & 23 & \\
\hline
\end{tabular}

Notes: †Hospital includes social facility for the elderly. In analysis of "residence", data of "dead" were eliminated. $* P<0.05$, Mann-Whitney $U$-test; $* * P<0.05$, chi-square test. Abbreviations: FIM, Functional Independence Measure; yr, years; M/F, male/female; $\mathrm{N}$, number of subjects.

inpatients was significantly longer than for middle-aged inpatients $(P<0.0001$, Mann-Whitney $U$-test $)$. FIM scores, including FIM gain and FIM efficiency, of elderly patients were also lower than those of middle-aged inpatients $(P<0.0001$, Mann-Whitney $U$-test $)$. There was a significant difference between the residence of elderly and middle-aged inpatients $(P<0.0001$, chi-squared test, home versus hospital). Importantly, we found that a significant number of elderly patients were transferred to other hospitals, including social care facilities, after discharge $(P<0.0001$, chi-squared test, admission versus discharge).

\section{Influence of sex on rehabilitation during hospital stay}

We examined the association between age and FIM scores in males and females separately. As shown in Figure 1, there was a significant negative correlation between FIM score at admission, discharge and FIM gain with age in males (FIM score at admission: $r=-0.24, P<0.001$; FIM score at discharge: $r=-0.35, P<0.001$; FIM gain: $r=-0.28$, $P<0.001)$. Similarly, there was a significant negative correlation between FIM score at admission, discharge and FIM gain with age in females (FIM score at admission: $r=-0.42$, $P<0.001$; FIM score at discharge: $r=-0.45, P<0.001$; FIM gain: $r=-0.21, P<0.001)$. Furthermore, we found there was a negative correlation between the length of hospital stay and age in males, but not in females (males: $r=-0.16, P=0.011$; females: $r=-0.048, P=0.30$ ). Next, we analyzed FIM gain to examine the difference of rehabilitation outcome between males and females (Table 2). Although there was a statistical difference in age between males and females (males:
A

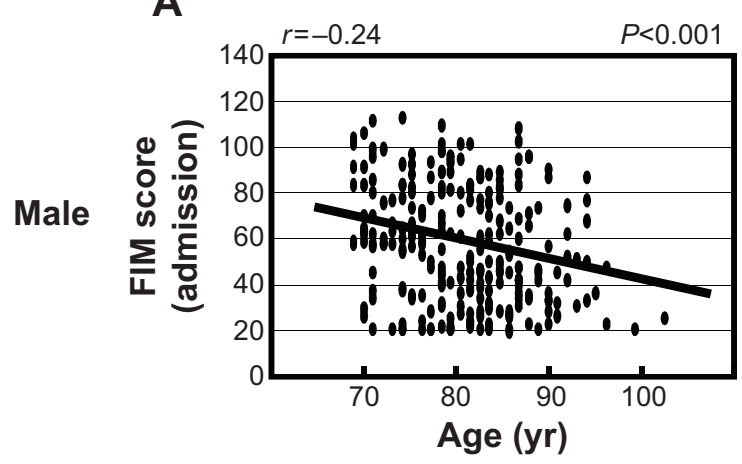

C

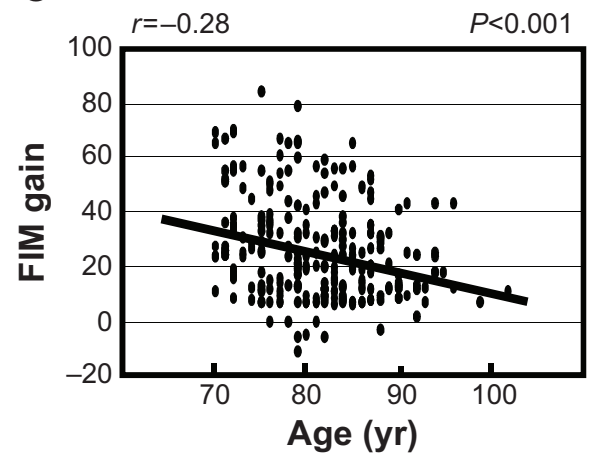

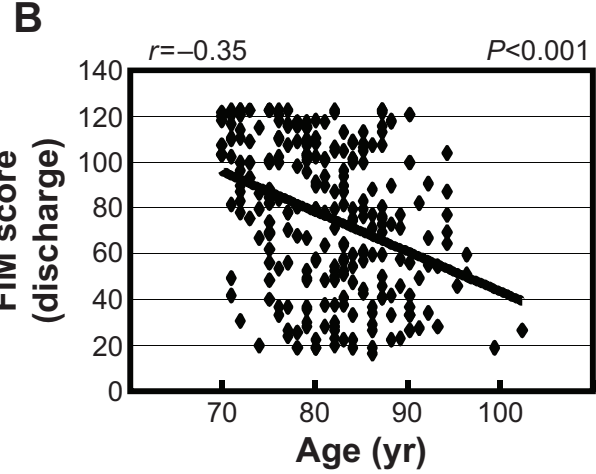

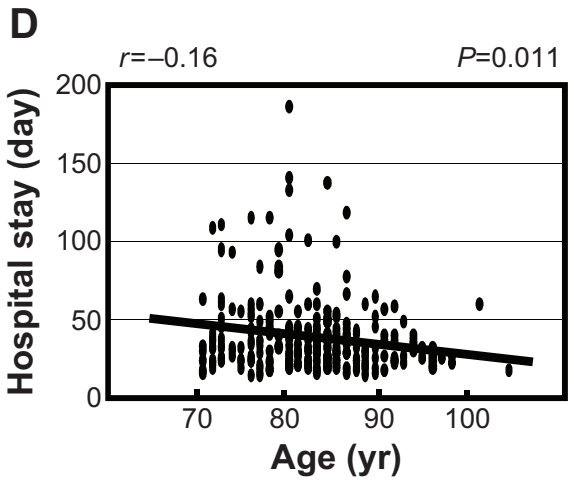

Figure I (Continued) 


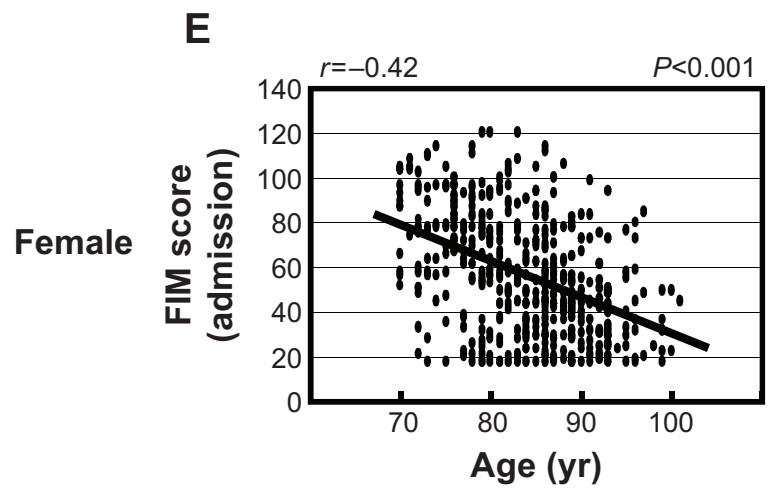

G

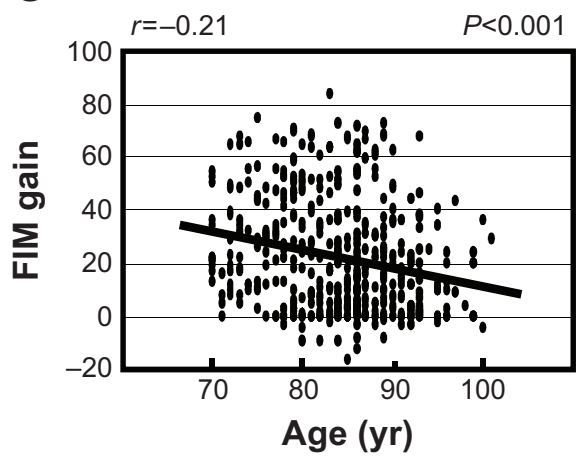

$\mathbf{F}$

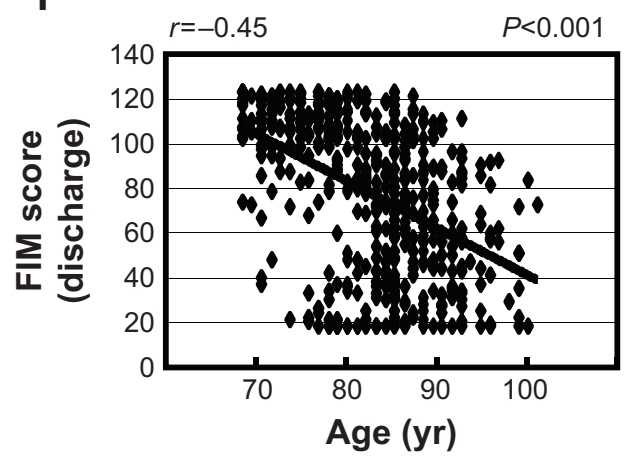

H

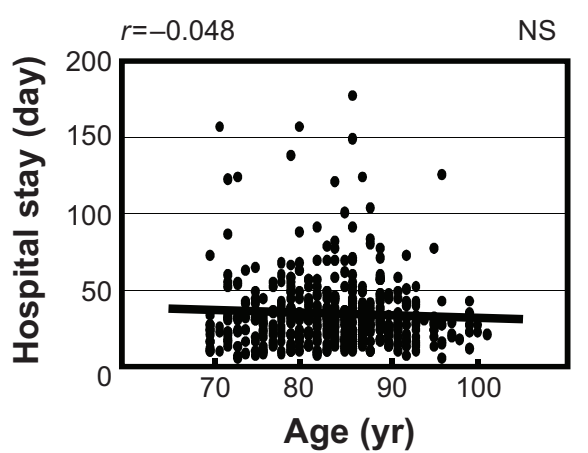

Figure I Association between age and FIM scores in males (A-D) and females (E-H).

Notes: FIM scores were measured at both hospital admission and discharge. FIM gain was calculated as the change in FIM score at hospital discharge. Length of hospital stay was counted as the total number of days spent in the hospital. The Spearman's rank correlation test was used for statistical analysis and $P$-values of $<0.05$ were considered statistically significant. FIM score at admission: panel (A) and (E); FIM score at discharge: panel (B) and (F); FIM gain: panel (C) and (G); length of hospital stay: panel (D) and (H).

Abbreviations: FIM, Functional Independence Measure; yr, years; NS, not significant.

$81.3 \pm 6.4$ years, $\mathrm{N}=256$; females: $83.8 \pm 6.8$ years, $\mathrm{N}=469$, $P<0.0001$, Mann-Whitney $U$-test) there were no significant differences between males and females in FIM gain or FIM efficiency (Mann-Whitney $U$-test, respectively).

\section{Influence of oral conditions and presence of disease on FIM scores}

The ability to close the lips and functional tongue movement were significant predictors of increased FIM gain, FIM efficiency, and the ratio of FIM improvement

Table 2 Comparison of sex in FIM score during hospital stay

\begin{tabular}{llll}
\hline & Male & Female & P-value \\
\hline $\mathrm{N}$ & 256 & 469 & \\
Age $(\mathrm{yr})$ & $81.3 \pm 6.4$ & $83.8 \pm 6.8$ & $<0.000 \mathrm{I}^{*}$ \\
Length of hospital stay (day) & $32.7 \pm 26.3$ & $33.3 \pm 23.4$ & 0.094 \\
FIM score & & & \\
$\quad$ At admission & $56.6 \pm 26.2$ & $56.3 \pm 26.0$ & 0.855 \\
$\quad$ At discharge & $77.7 \pm 33.7$ & $78.3 \pm 34.4$ & 0.813 \\
$\quad$ FIM gain & $21.2 \pm 19.9$ & $22.0 \pm 21.0$ & 0.992 \\
$\quad$ FIM efficiency & $0.92 \pm 1.1$ & $0.84 \pm 1.0$ & 0.338 \\
\hline
\end{tabular}

Note: $* P<0.05$, Mann-Whitney U-test.

Abbreviations: FIM, Functional Independence Measure; yr, years; N, number of subjects. after rehabilitation $(P<0.001$, Mann-Whitney $U$-test, respectively) (Table 3 ). On the other hand, there was no significant difference between stable or lost posterior occlusion (FIM gain: $P=0.129$, FIM efficiency: $P=0.0672$, ratio of FIM improvement: $P=0.646$, Mann-Whitney $U$-test). In addition, there were no significant differences in patients with an absence of teeth or presence of mobile teeth (absence of teeth, FIM gain: $P=0.375$, FIM efficiency: $P=0.653$, ratio of FIM improvement: $P=0.437$; presence of mobile teeth: FIM gain: $P=0.840$; FIM efficiency: $P=0.633$, ratio of FIM improvement: $P=0.588$, Mann-Whitney $U$-test, respectively). Of 256 elderly inpatients who had been previously diagnosed with a geriatric disease, 120 inpatients (46.9\%) had been diagnosed with cerebrovascular disease, 150 inpatients $(58.6 \%)$ with cognitive impairment, and 62 inpatients $(24.2 \%)$ with the acute phase of aspiration pneumonia. There was a significant negative correlation between the presence of cognitive impairment or aspiration pneumonia and FIM gain, FIM efficiency or ratio of FIM improvement $(P<0.001$, Mann-Whitney $U$-test, respectively). There was no significant difference in FIM scores between inpatients with or without cerebrovascular disease 
Table 3 Comparison of changes of FIM score during hospital stay

\begin{tabular}{|c|c|c|c|c|c|}
\hline Factor & Class & $\mathbf{N}(\mathbf{M} / \mathrm{F})$ & FIM gain & FIM efficiency & FIM improve (\%) \\
\hline \multirow[t]{3}{*}{ Posterior occlusion } & Stable & $203(72 /|3|)$ & $17.1 \pm 20.4$ & $0.668 \pm 0.95$ & $42.2 \pm 65.6$ \\
\hline & Loss & $59(29 / 30)$ & $12.7 \pm 17.8$ & $0.436 \pm 0.63$ & $37.7 \pm 56.5$ \\
\hline & & & $P=0.129$ & $P=0.0672$ & $P=0.646$ \\
\hline \multirow[t]{3}{*}{ Lips closure } & Closed & $222(82 / 140)$ & $17.8 \pm 20.4$ & $0.702 \pm 0.90$ & $20.7 \pm 41.4$ \\
\hline & Opened & $40(19 / 21)$ & $6.5 \pm 13.1$ & $0.133 \pm 0.65$ & $44.8 \pm 66.2$ \\
\hline & & & $P<0.000 I^{*}$ & $P<0.000 I^{*}$ & $P=0.002 *$ \\
\hline \multirow[t]{3}{*}{ Tongue movement } & Functional & $196(72 / 124)$ & $19.1 \pm 20.8$ & $0.73 I \pm 0.95$ & $47.8 \pm 68.5$ \\
\hline & Dysfunctional & $66(29 / 37)$ & $7.2 \pm 13.6$ & $0.273 \pm 0.58$ & $21.5 \pm 40.1$ \\
\hline & & & $P<0.000 I^{*}$ & $P<0.000 I^{*}$ & $P<0.0001 *$ \\
\hline \multirow[t]{3}{*}{ Teeth } & Remaining & $17 \mid(72 / 99)$ & $17.0 \pm 20.8$ & $0.642 \pm 0.97$ & $42.7 \pm 66.4$ \\
\hline & Edentulous & 9I (29/62) & $14.3 \pm 18.0$ & $0.565 \pm 0.70$ & $38.3 \pm 57.9$ \\
\hline & & & $P=0.375$ & $P=0.653$ & $P=0.437$ \\
\hline \multirow[t]{3}{*}{ Mobile teeth } & Presence & $39(19 / 20)$ & $15.8 \pm 22.7$ & $0.665 \pm 1.3$ & $51.8 \pm 95.7$ \\
\hline & None & $223(82 /|4|)$ & $16.1 \pm 19.4$ & $0.607 \pm 0.81$ & $39.3 \pm 56.2$ \\
\hline & & & $P=0.840$ & $P=0.633$ & $P=0.588$ \\
\hline \multirow[t]{3}{*}{ Cerebrovascular disease } & Presence & $120(50 / 70)$ & $13.8 \pm 18.4$ & $0.586 \pm 0.79$ & $36.5 \pm 56.5$ \\
\hline & None & $142(5|/ 9|)$ & $18.0 \pm 21.0$ & $0.640 \pm 0.97$ & $45.1 \pm 68.9$ \\
\hline & & & $P=0.0824$ & $P=0.329$ & $P=0.220$ \\
\hline Cerebrovascular disease + Loss & Presence & $30(13 / 17)$ & $8.1 \pm 14.8$ & $0.366 \pm 0.67$ & $27.9 \pm 56.1$ \\
\hline \multirow[t]{2}{*}{ of posterior occlusion } & None & $232(88 / / 44)$ & $17.1 \pm 20.3$ & $0.648 \pm 0.91$ & $42.9 \pm 64.4$ \\
\hline & & & $P=0.0073^{*}$ & $P=0.0199 *$ & $P=0.073$ \\
\hline \multirow[t]{3}{*}{ Cognitive impairment } & Presence & $150(55 / 95)$ & $12.1 \pm 17.9$ & $0.467 \pm 0.78$ & $37.5 \pm 62.8$ \\
\hline & None & $112(46 / 66)$ & $21.5 \pm 21.2$ & $0.814 \pm 0.98$ & $46.2 \pm 64.6$ \\
\hline & & & $P<0.000 I^{*}$ & $P<0.000 I^{*}$ & $P=0.009 *$ \\
\hline \multirow[t]{3}{*}{ Aspiration pneumonia } & Presence & $62(26 / 36)$ & $9.23 \pm 16.0$ & $0.330 \pm 0.59$ & $26.7 \pm 48.3$ \\
\hline & None & $200(75 / / 25)$ & $18.2 \pm 20.5$ & $0.704 \pm 0.95$ & $45.7 \pm 69.1$ \\
\hline & & & $P=0.0002^{*}$ & $P=0.000 I^{*}$ & $P=0.002 *$ \\
\hline
\end{tabular}

Note: $* P<0.05$, Mann-Whitney U-test (total $N=262$ ).

Abbreviations: FIM, Functional Independence Measure; M/F, male/female; N, number of subjects.

(FIM gain: $P=0.0824$, FIM efficiency: $P=0.329$, ratio of FIM improvement: $P=0.220$, Mann-Whitney $U$-test). Interestingly, there was a significant difference in FIM scores in patients who had both cerebrovascular disease and loss of posterior occlusion (FIM gain: $P=0.0073$, FIM efficiency: $P=0.0199$, Mann-Whitney $U$-test).

\section{ORs of factors predicting drastic improvement of the FIM score}

To further explore the significant factors which predicted drastic improvement of FIM scores, we defined elderly inpatients whose FIM gain was over 10 points as the "improved group", and calculated the ORs (Table 4). Stable posterior occlusion, the ability to close the lips, and functional tongue movement were significant factors predicting an improvement of FIM score (stable posterior occlusion: OR: 2.23, 95\% CI, 1.2-4.1, $P=0.008$; ability to close the lips: OR: 5.15, 95\% CI: 2.3-11.7, $P<0.0001$; functional tongue movement: OR: 5.74, 95\% CI: 3.0-11.0, $P<0.0001$ ), whereas absence of teeth or presence of mobile teeth were not associated with the improvement of FIM score. Furthermore, there was a significant negative correlation between the presence of cognitive impairment or aspiration pneumonia and the improvement of FIM score (cognitive impairment: OR: 0.29, 95\% CI, 0.17-0.49, $P<0.0001$; aspiration pneumonia: OR: $0.27,95 \%$ CI: $0.15-0.51, P<0.0001$ ), whereas there was no correlation between presence of cerebrovascular disease and improvement of FIM score. Interestingly, there was a significant negative correlation between the combination of cerebrovascular disease and loss of posterior occlusion and the improvement of FIM scores (OR: 0.31, 95\% CI: $0.13-0.73, P=0.005)$.

\section{Influence of various factors on BMI}

We used BMI to investigate the association between nutritional status and other factors in elderly inpatients. As shown in Figure 2, there was no significant correlation between BMI and age in elderly inpatients $(r=0.05, P=0.42)$. There was also no significant difference in BMI value between males and females $(P=0.67$, Mann-Whitney $U$-test). However we found that the BMI was significantly higher in patients with stable posterior occlusions and functional 
Table 4 Odds ratio of various factors inducing improvement of FIM score

\begin{tabular}{|c|c|c|c|}
\hline Factor & Odds ratio & $95 \% \mathrm{Cl}$ & $P$-value \\
\hline Stable posterior occlusion & 2.23 & I.2 4.1 & $0.008^{*}$ \\
\hline Closed lips & 5.15 & $2.3 \sim 11.7$ & $<0.000 I^{*}$ \\
\hline Functional tongue movement & 5.74 & $3.0 \sim 11.0$ & $<0.0001 *$ \\
\hline Remaining teeth & 1.16 & $0.70 \sim 1.9$ & 0.57 \\
\hline Presence of mobile teeth & 1.16 & $0.58 \sim 2.3$ & 0.68 \\
\hline Presence of cerebrovascular disease & 0.65 & $0.40 \sim 1.1$ & 0.09 \\
\hline $\begin{array}{l}\text { Presence of cerebrovascular disease }+ \text { Loss } \\
\text { of posterior occlusion }\end{array}$ & 0.31 & $0.13 \sim 0.73$ & $0.005^{*}$ \\
\hline Presence of cognitive impairment & 0.29 & $0.17 \sim 0.49$ & $<0.000 I^{*}$ \\
\hline Presence of aspiration pneumonia & 0.27 & $0.15 \sim 0.5 \mathrm{I}$ & $<0.000 I^{*}$ \\
\hline
\end{tabular}

Notes: $* P<0.05$, chi-square test. Over 10 points of FIM gain during hospital stay were extracted as "improvement".

Abbreviations: FIM, Functional Independence Measure; $\mathrm{Cl}$, confidence interval.

tongue movement (posterior occlusion: stable versus loss, $P=0.046$; tongue movement: functional versus dysfunctional, $P=0.027$, Mann-Whitney $U$-test). There were no significant differences between BMI and other oral factors such as the ability to close the lips, absence of teeth or presence of mobile teeth. In addition, the BMI of patients with either cognitive impairment or aspiration pneumonia were significantly lower (cognitive impairment: $P=0.023$; aspiration pneumonia: $P=0.005$, Mann-Whitney $U$-test); however, there was no significant difference of BMI between patients with or without cerebrovascular disease. In the "improved group", BMI value, but not age, was significantly higher (BMI: FIM gain $>10$ points versus FIM gain $<10$ points, $P=0.0002$; age: FIM gain $>10$ points versus FIM gain $<10$ points, $P=0.562$, Mann-Whitney $U$-test).

\section{Discussion}

In general, risk factors that hamper the elderly from leading a healthy life include various physical, psychological, and social problems occurring in older age, as well as a higher incidence of disease. ${ }^{13,14}$ In order to effectively practice the multidisciplinary care needed to support geriatric treatment, development of a screening or examination system to evaluate the success of such care is needed. Aging is one of the factors correlated with diminished physical recovery, particularly in extremely elderly patients. ${ }^{13}$ As shown in Table 1, performance of ADLs by elderly inpatients was significantly diminished, and rehabilitation of elderly inpatients was much less effective when compared with middle-aged patients. These results demonstrate that the functional recovery of
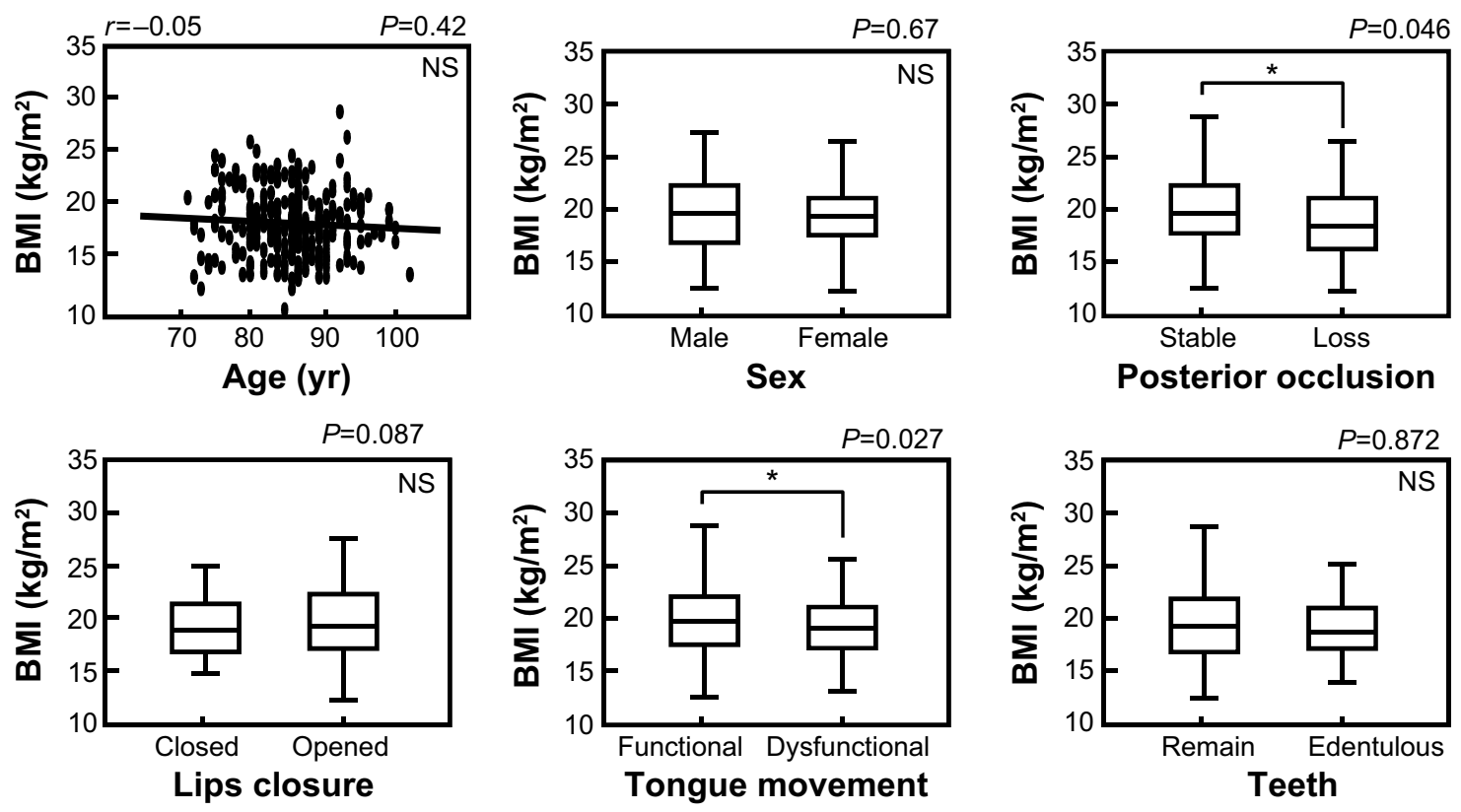

Figure 2 (Continued) 

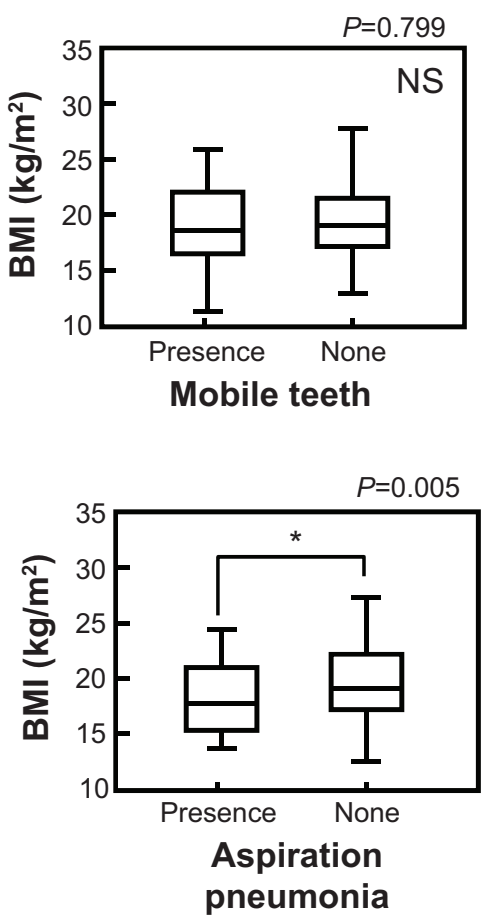
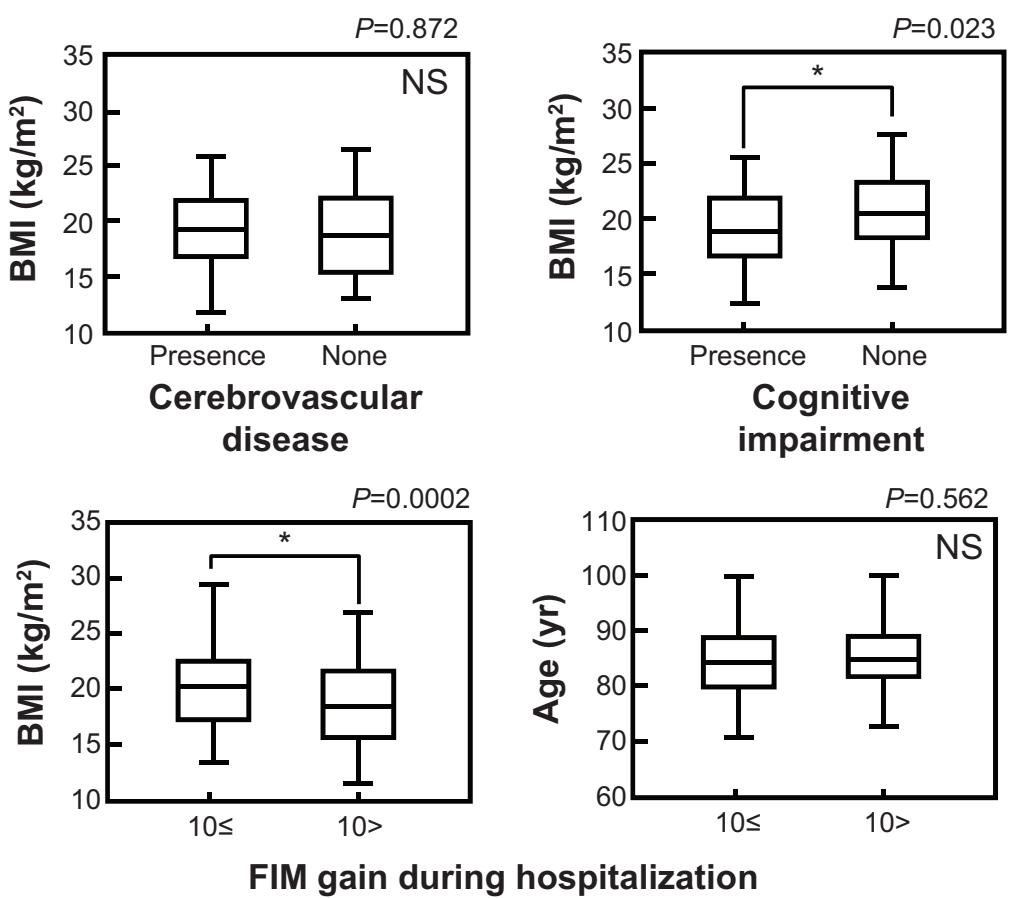

Figure 2 Association between BMI values and other factors.

Notes: Data were described by the mean \pm standard deviation (SD). FIM gain >10 points: $\mathrm{N}=133$ (male/female: $48 / 85$ ); FIM gain <I0 points: $\mathrm{N}=129$ (male/female: $53 / 76$ ). The Mann-Whitney $U$-test was used for statistical analysis and $* P$-values of $<0.05$ were considered statistically significant.

Abbreviations: FIM, Functional Independence Measure; NS, not significant; BMI, body mass index; yr, years.

elderly inpatients is difficult and must be supported by proper treatment and possibly nursing care. In addition, there was a significant negative correlation between aging and FIM scores at the time of admission and discharge, as well as FIM gain during hospitalization. Greenwald et al has reported previously that no sex-related differences were observed in FIM scores, including FIM efficiency, on admission and discharge. ${ }^{15}$ Similarly, we demonstrated that there was no association between sex and the functional outcome of elderly inpatients after rehabilitation. In elderly males, there was a negative correlation between length of stay and age; further analysis is needed to clarify this difference between males and females.

Predicting the functional outcome in elderly inpatients during rehabilitation may have a significant impact on the decision of discharge destination. ${ }^{16}$ Therefore, we explored several factors, such as oral condition, to predict whether performance of ADLs is improved by standard rehabilitation in elderly inpatients. As shown in Table 3, the ability to close the lips and functional tongue movement, which relates to oral muscle strength, were significant predictors of an improvement in FIM scores. On the other hand, neither the loss of teeth nor presence of mobile teeth had any correlation with a change in FIM scores. Although the presence of mobile teeth is one of the important contributors of chronic infectious periodontitis, ${ }^{17}$ we hypothesize that functional oral muscles, but not oral infection caused by periodontitis, may contribute more to the improvement of ADL performance. Functional oral muscles including the orbicularis oris muscle and tongue muscle may lead to improvement of the systemic physical strength. In addition, although we considered the scale values of FIM as a linear progression, a patient who starts from a lower value of FIM has more opportunity to recover points compared to those who start from very high values. Therefore, it might be useful to consider the recovery of expressing points of FIM using percentage values. Interestingly, we found that the same tendency was observed between FIM gain and the ratio of FIM improvement (\%) as shown in Table 3. It is important to note that, in the present study, the determination of the presence of teeth included nonfunctional teeth, such as the stump of the tooth, and should not to be mistaken as proper occlusion. Furthermore, we demonstrated that the ability to close the lips and functional tongue movement were highly significant predictors of improved FIM score, whereas loss of teeth or presence of mobile teeth were not correlated with any improvement. In addition, we also showed that the stability of the posterior occlusion was a significant predictor of a major improvement 
in FIM score, although there was no significant difference between stability and loss of posterior occlusion in changes of FIM scores. These results indicate that a stable posterior occlusion is needed for improvement of ADL performance after rehabilitation in elderly inpatients. Importantly, there was no statistical difference in age between the "improved group" and patients whose FIM gain was $>1$ point $(P=0.099$, Mann-Whitney $U$-test, FIM gain $>10$ points: $81.9 \pm 6.9$ years, $\mathrm{N}=463$; FIM gain $>10$ points: $82.6 \pm 6.8$ years, $\mathrm{N}=616$ ). These findings support the clinical usefulness of oral examination and collaboration with a dentist for elderly inpatients undergoing rehabilitation.

In general, a significant proportion of elderly inpatients have additional geriatric diseases such as cerebrovascular disease, which may include severe cognitive decline. ${ }^{13,14}$ Symptoms are characterized by confusion and/or stupor, which are difficult to manage clinically and require a great deal of care, because the diseases hamper ADL performance in elderly inpatients. ${ }^{18}$ It has been reported previously that no significant differences were found between spinal cord injury and rehabilitation discharge. ${ }^{15}$ In the present study there was a significant negative correlation between the presence of aspiration pneumonia and improvement of ADL performance as shown in Table 3. Interestingly, we found that both the presence of cerebrovascular disease and loss of posterior occlusion together significantly reduced improvement of FIM scores during rehabilitation, despite no significant differences observed in either factor independently. These data suggest that the examination of the posterior occlusion should not be ignored to predict the effectiveness of rehabilitation in elderly inpatients. Additionally, both Tables 3 and 4 show that presence of cognitive impairment may be an important predictor of the improvement of FIM scores. This supports our observation that motivation in elderly inpatients is important for the improvement of ADL performance.

Lin et al had previously reported that maintenance of proper nutritional status in the elderly is essential to reduce hospitalization, death risk, and medical expenses, and improves the quality of care. ${ }^{19}$ Other epidemiological studies have also shown that weight loss, reduced caloric intake, and the reduced intake of specific nutrients are associated with the impairment of physical function in the elderly. ${ }^{20,21}$ To confirm these associations, we investigated the relation between nutritional status and other factors, such as oral condition, in elderly inpatients using BMI. While there was no correlation between BMI and either age or sex, we found that there was a significant difference in BMI in patients with a stable posterior occlusion and functional tongue movement, but no difference when looking at the ability to close the lips, loss of teeth or presence of mobile teeth. This is not surprising because both a stable posterior occlusion and functional tongue movement are critical for proper eating. Furthermore, although the BMI of patients with either cognitive impairment or aspiration pneumonia were significantly lower, there was no significant correlation between low BMI and cerebrovascular disease. With severe cerebrovascular disease there is a high risk of malnutrition, although in the present study, significant correlation between BMI and presence of cerebrovascular disease was not observed, possibly due to the lack of discrimination between mild and severe cases. Further analysis will be needed in the future to better clarify any association between cerebrovascular disease and BMI. Previously, it has been reported that both a mini nutritional assessment and measurement of serum albumin levels can predict the rehabilitation outcome of elderly inpatients. ${ }^{22}$ Our findings also demonstrate that the BMI of patients who had an FIM gain greater than 10 points was significantly higher than patients who had a lesser increase. The risk of malnutrition may rise in the majority of chronic illnesses due to physical dependency and impairment of oral condition in the elderly; therefore, nutritional management is important to improve ADL performance and the quality of life.

The present study had several limitations. First, because all patients of this series were assessed at our hospital, this study is inherently limited by single-center selection bias. Therefore, the results may not reflect the actual situation of the elderly in every country. Second, BMI is not always reflective of malnutrition in elderly inpatients; very elderly people tend to have very low BMIs. Third, we did not assess the comorbidities of diseases, although comorbidities may confound other factors and also affect rehabilitation effectiveness. Fourth, evaluation of the ability to close the lips may be inaccurate; often it is difficult to elucidate the root cause of this inability, which may include impairment of oral muscles and/or respiratory difficulty caused by acute phases of geriatric diseases. Finally, although our estimation models were adjusted for potential factors such as age and sex, no adjustments were made for other factors which may impact ADL performance such as cancer chemotherapy, medication, smoking, etc. We believe that risk adjustment approach could increase the validity of this study in the future.

\section{Conclusion}

In the present study, we found that age, malnutrition, and disorders of oral function are significant factors which may predict an improvement in ADL performance of 
elderly inpatients after rehabilitation in the hospitalization. From these results, evaluation of elderly patients by multidisciplinary rehabilitation teams may better predict the effectiveness of rehabilitation and destination of inpatients after discharge.

\section{Acknowledgment}

The authors thank the medical staff of Tottori Municipal Hospital for their assistance with database management.

\section{Author contributions}

All authors made substantial contributions to conception and design, acquisition of data, or analysis and interpretation of data; took part in either drafting the article or revising it critically for important intellectual content; gave final approval of the version to be published.

\section{Disclosure}

We have no support or funding to report. We have no conflict of interest in the present study.

\section{References}

1. Arai H, Ouchi Y, Yokode M, et al. Toward the realization of a better aged society: messages from gerontology and geriatrics. Geriatr Gerontol Int. 2012;12(1):16-22.

2. Igaki $\mathrm{H}$, Onishi $\mathrm{H}$, Nakagawa K, et al. A newly introduced comprehensive consultation fee in the national health insurance system in Japan: a promotive effect of multidisciplinary medical care in the field of radiation oncology - results from a questionnaire survey. Jpn J Clin Oncol. 2013; 43(12):1233-1237.

3. Alagiakrishnan K, Bhanji RA, Kurian M. Evaluation and management of oropharyngeal dysphagia in different types of dementia: a systematic review. Arch Gerontol Geriatr. 2013;56(1):1-9.

4. van der Maarel-Wierink CD, Vanobbergen JN, Bronkhorst EM, Schols JM, de Baat C. Meta-analysis of dysphagia and aspiration pneumonia in frail elders. $J$ Dent Res. 2011;90(12):1398-1404.

5. Deschodt M, Flamaing J, Haentjens P, Boonen S, Milisen K. Impact of geriatric consultation teams on clinical outcome in acute hospitals: a systematic review and meta-analysis. BMC Med. 2013;11:48.

6. Nakayama E, Tohara H, Hino T, et al. The effects of ADL on recovery of swallowing function in stroke patients after acute phase. J Oral Rehabil. Epub 2014 Jul 8.
7. Barnes DE, Mehta KM, Boscardin WJ, et al. Prediction of recovery, dependence or death in elders who become disabled during hospitalization. J Gen Intern Med. 2013;28(2):261-268.

8. Takata Y, Ansai T, Soh I, et al. High-level activities of daily living and disease-specific mortality during a 12-year follow-up of an octogenarian population. Clin Interv Aging. 2013;8:721-728.

9. Granger CV, Deutsch A, Russell C, Black T, Ottenbacher KJ. Modifications of the FIM instrument under the inpatient rehabilitation facility prospective payment system. Am J Phys Med Rehabil. 2007;86(11): 883-892.

10. Galloway RV, Granger CV, Karmarkar AM, et al. The Uniform Data System for Medical Rehabilitation: report of patients with debility discharged from inpatient rehabilitation programs in 2000-2010. Am J Phys Med Rehabil. 2013;92(1):14-27.

11. Stineman MG, Escarce JJ, Tassoni CJ, Goin JE, Granger CV, Williams SV. Diagnostic coding and medical rehabilitation length of stay: their relationship. Arch Phys Med Rehabil. 1998;79(3):241-248.

12. Moyle W, Murfield JE, O'Dwyer S, Van Wyk S. The effect of massage on agitated behaviours in older people with dementia: a literature review. J Clin Nurs. 2013;22(5-6):601-610.

13. Langlois F, Vu TT, Chassé K, Dupuis G, Kergoat MJ, Bherer L. Benefits of physical exercise training on cognition and quality of life in frail older adults. J Gerontol B Psychol Sci Soc Sci. 2013;68(3): 400-404.

14. Brown CJ, Flood KL. Mobility limitation in the older patient: a clinical review. JAMA. 2013;310(11):1168-1177.

15. Greenwald BD, Seel RT, Cifu DX, Shah AN. Gender-related differences in acute rehabilitation lengths of stay, charges, and functional outcomes for a matched sample with spinal cord injury: a multicenter investigation. Arch Phys Med Rehabil. 2001;82(9):1181-1187.

16. Messina G, Lorena R, Chiara B, Emma C, Cecilia Q, Nicola N. Which Factors Influence Functional Patients Improvements During Rehabilitation? Glob J Health Sci. 2014;6(3):74-81.

17. Armitage GC. Clinical evaluation of periodontal diseases. Periodontol 2000. 1995;7:39-53

18. Caillet P, Laurent M, Bastuji-Garin S, et al. Optimal management of elderly cancer patients usefulness of the Comprehensive Geriatric Assessment. Clin Interv Aging. 2014;9:1645-1660.

19. Lin SJ, Hwang SJ, Liu CY, Lin HR. The relationship between nutritional status and physical function, admission frequency, length of hospital stay, and mortality in old people living in long-term care facilities. J Nurs Res. 2012;20(2):110-121.

20. Lin MR, Wolf SL, Hwang HF, Gong SY, Chen CY. A randomized, controlled trial of fall prevention programs and quality of life in older fallers. J Am Geriatr Soc. 2007;55(4):499-506.

21. Bates CJ, Benton D, Biesalski HK, et al. Nutrition and aging: a consensus statement. J Nutr Health Aging. 2002;6(2):103-116.

22. Ülger Z, Halil M, Kalan I, et al. Comprehensive assessment of malnutrition risk and related factors in a large group of community-dwelling older adults. Clin Nutr. 2010;29(4):507-511.
Clinical Interventions in Aging

\section{Publish your work in this journal}

Clinical Interventions in Aging is an international, peer-reviewed journal focusing on evidence-based reports on the value or lack thereof of treatments intended to prevent or delay the onset of maladaptive correlates of aging in human beings. This journal is indexed on PubMed Central, MedLine,

\section{Dovepress}

CAS, Scopus and the Elsevier Bibliographic databases. The manuscript management system is completely online and includes a very quick and fair peer-review system, which is all easy to use. Visit http://www.dovepress. com/testimonials.php to read real quotes from published authors. 